\title{
Calidad de vida: Una revisión teórica del concepto
}

\author{
Quality of life: A theoretical review
}

\author{
Alfonso Urzúa M. \\ Escuela de Psicología, Universidad Católica del Norte, Chile \\ $\&$ \\ Alejandra Caqueo-Urízar \\ Depto. de Filosofía y Psicología, Universidad de Tarapacá, Chile
}

(Rec: 10 de noviembre de 2011 / Acep: 10 de enero de 2012)

\begin{abstract}
Resumen
El concepto de calidad de vida ha comenzado a utilizarse cada vez más en el campo de las evaluaciones en salud o como medida de bienestar. Pese a esto, no existe una definición única del concepto ni una completa diferenciación con otros conceptos similares, siendo frecuentemente mal utilizado. El presente trabajo presenta una revisión teórica del concepto, así como una clasificación de los distintos modelos de definiciones, incluyendo una propuesta de modelo centrado en la evaluación cognitiva. Se desarrollan también los principales problemas de la investigación en el área de la calidad de vida.
\end{abstract}

Palabras clave: Calidad de vida, concepto.

\begin{abstract}
The concept of quality of life has begun to be used increasingly in the field of health assessments or as a measure of well-being. Despite this, there is no single definition or a complete difference from other similar concepts often being misused. This paper presents a theoretical concept and a classification of the different models of definitions, including a proposed model based on cognitive assessment. They also develop major problems confronting research in the area of quality of life.
\end{abstract}

Keywords: Quality of life, concept. 


\section{Introducción}

La utilización del concepto de Calidad de Vida (CV) puede remontarse a los Estados Unidos después de la Segunda Guerra Mundial, como una tentativa de los investigadores de la época de conocer la percepción de las personas acerca de si tenían una buena vida o si se sentían financieramente seguras (Campbell, 1981; Meeberg, 1993).

Su uso extendido es a partir de los sesentas, cuando los científicos sociales inician investigaciones en $\mathrm{CV}$ recolectando información y datos objetivos como el estado socioeconómico, nivel educacional o tipo de vivienda, siendo muchas veces estos indicadores económicos insuficientes (Bognar, 2005), dado que sólo eran capaces de explicar un $15 \%$ de la varianza en la CV individual.

Frente a esto, algunos psicólogos plantearon que mediciones subjetivas podrían dar cuenta de un mayor porcentaje de varianza en la $\mathrm{CV}$ de las personas, toda vez que indicadores psicológicos como la felicidad y la satisfacción explicaban sobre un 50\% de la varianza (Campbell \& Rodgers, 1976; Haas, 1999).

Para algunos autores, el concepto de CV se origina a fin de distinguir resultados relevantes para la investigación en salud, derivado de las investigaciones tempranas en bienestar subjetivo y satisfacción con la vida (Smith, Avis \& Assman, 1999).

El hecho que desde sus inicios haya estado vinculado a otras variables psicológicas que involucran en sí mismas el concepto de bienestar, ha posibilitado que, a la fecha, aún muchos investigadores no diferencien claramente en sus estudios cada concepto o lo utilicen de manera indistinta. Tal como lo comenta Meeberg (1993), muchos otros términos como satisfacción con la vida, bienestar subjetivo, bienestar, autoreporte en salud, estado de salud, salud mental, felicidad, ajuste, estado funcional y valores vitales son usados para designar $\mathrm{CV}$, agregando además que muchos autores no definen lo que para ellos significa $\mathrm{CV}$, introduciendo confusión acerca de lo que intentan referir.

Datos complementarios a esta idea son proporcionados por Gill \& Fenstein (1994), quienes, en una revisión de artículos sobre $\mathrm{CV}$, encuentran que sólo un $35 \%$ de los modelos evaluados tenían un desarrollo conceptual complejo, cerca de un $25 \%$ de los autores no definía CV y más del $50 \%$ de los modelos no diferenciaba entre CV y los factores que la influencian. Reportes similares son informados por Taillefer, Dupuis, Roberge \& Lemay (2003), quienes en una revisión sobre modelos existentes en la literatura sobre $\mathrm{CV}$, plantean que desde que este concepto comenzó a atraer el interés de los investigadores en los años 60 , ha habido un rápido incremento en su uso, el cual ha evidenciado problemas tanto en la definición del concepto, las dimensiones que le comprenden, su medición y los factores que la pueden influenciar.

Esta gran dispersión y diversidad de concepciones puede derivar de la naturaleza diversa del concepto (Cummins, 2000) o bien a que este es un término multidisciplinario (Haas, 1999). Así, los economistas tenderán a centrar su medición en elementos tangibles de la CV y, por otro lado, los científicos sociales lo harán en la percepción individual sobre la CV. El concepto de CV actualmente incorpora tres ramas de las ciencias: economía, medicina y ciencias Sociales. Cada una de estas disciplinas ha promovido el desarrollo de un punto de vista diferente respecto a cómo debiera ser conceptualizada la CV (Cummins, 2004).

En la Tabla 1 se encuentran definiciones aportadas por diversos autores al referirse a la $\mathrm{CV}$.

Tabla 1. Diversas definiciones para el concepto de calidad de vida.

\begin{tabular}{|c|c|}
\hline Referencia & Definición Propuesta \\
\hline Ferrans (1990b) & $\begin{array}{l}\text { Calidad de vida general definida como el bienestar personal derivado de la satisfacción o insatisfacción con } \\
\text { áreas que son importantes para él o ella. }\end{array}$ \\
\hline Hornquist (1982) & $\begin{array}{l}\text { Define en términos de satisfacción de necesidades en las esferas física, psicológica, social, de actividades, } \\
\text { material y estructural. }\end{array}$ \\
\hline Shaw (1977) & $\begin{array}{l}\text { Define la calidad de vida de manera objetiva y cuantitativa, diseñando una ecuación que determina la calidad } \\
\text { de vida individual: } Q \mathrm{QL}=\mathrm{NE} \text { x }(\mathrm{H}+\mathrm{S}) \text {, en donde NE representa la dotación natural del paciente, } \mathrm{H} \text { la contri- } \\
\text { bución hecha por su hogar y su familia a la persona y } \mathrm{S} \text { la contribución hecha por la sociedad. Críticas: la } \\
\text { persona no evalúa por sí misma, segundo, no puede haber cero calidad de vida. }\end{array}$ \\
\hline Lawton (2001) & $\begin{array}{l}\text { Evaluación multidimensional, de acuerdo a criterios intrapersonales y socio-normativos, del sistema personal } \\
\text { y ambiental de un individuo. }\end{array}$ \\
\hline Haas (1999) & $\begin{array}{l}\text { Evaluación multidimensional de circunstancias individuales de vida en el contexto cultural y valórico al } \\
\text { que se pertenece. }\end{array}$ \\
\hline Bigelow et al., (1991) & Ecuación en donde se balancean la satisfacción de necesidades y la evaluación subjetiva de bienestar. \\
\hline Calman (1987) & $\begin{array}{l}\text { Satisfacción, alegría, realización y la habilidad de afrontar... medición de la diferencia, en un tiempo, entre } \\
\text { la esperanza y expectativas de una persona con su experiencia individual presente. }\end{array}$ \\
\hline Martin \& Stockler (1998) & Tamaño de la brecha entre las expectativas individuales y la realidad a menor intervalo, mejor calidad de vida. \\
\hline Opong et al., (1987) & Condiciones de vida o experiencia de vida. \\
\hline
\end{tabular}




\section{Categorías de definiciones}

Tal como se ha visto, no existen definiciones únicas sobre el concepto de $\mathrm{CV}$, sin embargo, con fines didácticos estas definiciones pueden ser agrupadas en distintas categorías. Las tres primeras que se revisarán corresponden a una categorización (Borthwick-Duffy, 1992; Felce \& Perry, 1995).

\section{a) $C V=$ Condiciones de vida}

Bajo esta perspectiva, la CV es equivalente a la suma de los puntajes de las condiciones de vida objetivamente medibles en una persona, tales como salud física, condiciones de vida, relaciones sociales, actividades funcionales $\mathrm{u}$ ocupación. Este tipo de definición permitiría comparar a una persona con otra desde indicadores estrictamente objetivos, sin embargo, pareciera que estos apuntan más una cantidad que CV (Hollanflsworth, 1988).

Definiciones de este tipo conllevan diversos problemas. Por una parte, no existen parámetros universales de una buena u óptima $\mathrm{CV}$, ya que los estándares con los que se evalúa son distintos dependiendo de los individuos y de la sociedad. Por otra parte, y tal como lo menciona Edgerton (1990), existiría independencia entre condiciones de vida y eventos subjetivos de bienestar, tal como lo demuestran los análisis sobre la cantidad de varianza explicada por las condiciones objetivas de vida y a las constantes constataciones que los países con mayor nivel de ingresos no necesariamente tienen los mayores niveles de CV cuando ésta es evaluada por los propios ciudadanos. Además, el bienestar global no sólo dependería de los recursos propios sino también del ambiente socio político del cual el bienestar personal es nutrido (Shen \& Lai, 1998). Para Lefley (1998), la CV es un concepto imbuido de significado cultural, económico y político. El producto final de cualquier modelo de CV sería un estado global de bienestar ligado a determinados puntos de vista, el cual parte de los supuestos que todos los individuos tendrían derecho a un estado de bienestar y además que esta experiencia de bienestar puede ser medida en términos de indicadores universales objetivos.

O'Boyle (1994) plantea que la evaluación objetiva asume que la salud, ambiente físico, ingresos, vivienda y otros indicadores observables y cuantificables son mediciones válidas de $\mathrm{CV}$ y son estándares, sin embargo, la vinculación entre estos criterios objetivos y la CV percibida sería compleja y difícil de predecir.

\section{b) $C V=$ Satisfacción con la vida}

Bajo este marco, se considera a la CV como un sinónimo de satisfacción personal. Así, la vida sería la sumatoria de la satisfacción en los diversos dominios de la vida.

Este tipo de definiciones también tiene sus detractores, ya que al otorgar el principal foco de interés al bienestar subjetivo y dejar de lado las condiciones externas de vida, supone un individuo capaz de abstraerse, por ejemplo, de su contexto económico, social, cultural, político o incluso de su propio estado de salud, para lograr la satisfacción personal.

Sin embargo, a pesar de que se reconoce que la satisfacción es una evaluación individual donde el marco de referencia es personal, no es posible desconocer que esta evaluación es afectada por la experiencia y el juicio de una persona en una determinada situación.

Un punto interesante de discutir y que también lleva relación con la evaluación netamente subjetiva de la $\mathrm{CV}$, es el dilema de la "conciencia", "insight" o grado de "introspección" necesarios para una auto evaluación, ya que esta afectaría necesariamente los parámetros y estándares con que la persona se autoevaluaría.

Este foco de discusión se ha focalizado principalmente, en el campo del estudio de la $\mathrm{CV}$, en pacientes con problemas mentales, en especial en el de las personas viviendo con esquizofrenia, trastornos bipolares o retardo mental (Verdugo, Schalock, Keith \& Stancliffe, 2005). La inclusión del autoreporte de la CV percibida además de los indicadores objetivos tradicionales ha sido objeto de amplias discusiones. La validez de la incorporación de una perspectiva subjetiva de los pacientes en la medición de CV es apoyada por parte de la literatura psiquiátrica en el campo de la auto evaluación de su estado actual, funcionamiento y habilidades que deriven placer de las actividades cotidianas (Bishop, Walling, Dott, Folies \& Bucy, 1999; Caqueo \& Lemos, 2008; Caqueo-Urízar, Gutiérrez-Maldonado \& Miranda-Castillo; 2009), empero, detractores sugieren que la medición en pacientes psiquiátricos debería ser distinta a los otros campos de la medicina debido a factores como la introspección.

Al respecto, Jenkins (1992) plantea que las respuestas de los pacientes con enfermedad mental pueden reflejar idealmente su situación actual física, psicológica y social y que estas respuestas pueden estar mediadas por expectativas individuales, deseos personales o estado mental, pudiendo en esta evaluación surgir errores frecuentes por distorsiones perceptuales, carencia de insight, ilusiones y respuestas prejuiciadas por las limitaciones del uso de metodologías de autorespuesta. Jenkins concluye que la evaluación en CV debe estar basada en fenómenos observables o descriptivos específicos, teniendo cuidado con el uso de las ponderaciones personales, los cuales pueden ser prejuiciados por la medicación, funcionamiento cognitivo o emocional y motivaciones por mejorar su vida. Deben también considerarse las aspiraciones y la capacidad para darse cuenta de las personas, ya que los pacientes mentales pueden carecer de insigth o motivación para mejorar su vida. A menos que el paciente reconozca déficit en áreas de su vida y exhiba motivación a cambiar, la CV será alta.

A menudo, los pacientes que no han hecho insight sobre el impacto de la enfermedad en sus vidas tienden a tener puntajes más elevados que aquellos que sí han hecho insight. 
Esta discusión, que pareciera estar ajena al campo de la evaluación de la CV en personas sin patologías mentales, puede ser significativa si se considera el supuesto que no todas las personas tienen la capacidad de "darse cuenta" o hacer insight con relación a su propia existencia, hecho sugerido por algunos sectores, como por ejemplo los educadores populares, quienes trabajan en gatillar el despertar de la gente a la realidad, a fin de que asuman responsablemente la conducción, autogestión y autodeterminación de sus vidas.

Algunos autores sugieren acotar el término adjetivizándolo, proponiendo la denominación CV subjetiva, definida como el grado individual de satisfacción con la vida, de acuerdo a estándares internos o referentes. Este término debe distinguirse de las evaluaciones cognitivas, como la satisfacción con la vida y las evaluaciones afectivas, como lo es la felicidad (Shen \& Lai, 1998), ya que si se mide la CV general en términos de la satisfacción o felicidad reportada, se está asumiendo que la gente hace el mejor juicio de lo que es mejor para su vida y que tiene conciencia de lo que hace incrementar o decaer su CV (Michalos, 2004). Pese a esto, para algunos investigadores las mediciones de felicidad pueden ser un importante camino hacia la operacionalización de la variedad de ideas referidas por la frase "calidad de vida", porque esta estaría a la base de la satisfacción personal en los distintos dominios de la vida (Michalos, 2004).

\section{c) $C V=($ Condiciones de vida + Satisfacción con la vida)}

Desde esta perspectiva, las condiciones de vida pueden ser establecidas objetivamente mediante indicadores biológicos, sociales, materiales, conductuales y psicológicos, los que sumados a los sentimientos subjetivos sobre cada área pueden ser reflejados en el bienestar general. Este grupo de definiciones intentaría conciliar la discusión acerca de considerar a la CV desde un punto de vista netamente objetivo o desde uno subjetivo. Un punto importante a destacar es el que toma la significación de cada una de las evaluaciones, tanto objetivas como subjetivas, en cada dominio de vida en particular, ya que estas son interpretables sólo en relación con el lugar de importancia que toma en la persona (Felce \& Perry, 1995). Para algunos autores, la CV global tendría más significado si se la considera como compuesta por los sentimientos de satisfacción frente a diversos aspectos de la vida (Shen \& Lai, 1998).
Discusiones similares sobre la CV objetiva o subjetiva son encontradas con frecuencia en la literatura; al respecto, Cummins (2000), luego de un extenso análisis sobre lo subjetivo y lo objetivo en la mediciones en $\mathrm{CV}$, concluye que ambos tipos de medidas han demostrado ser indicadores útiles, y aun cuando son independientes, el grado de dependencia aumenta cuando las condiciones objetivas de vida son más pobres. Sobre lo mismo, Wrosch \& Scheier (2003) y Huppert \& Whittington (2003) plantean que se ha investigado la $\mathrm{CV}$ relacionándola con indicadores objetivos (condiciones de vida, estado de salud) e indicadores subjetivos (satisfacción con la vida, bienestar). Ambos asumen que explican parte de la varianza de la CV reportada por las personas y que las personas pueden valorar más una cosa que otra, dependiendo de la significación que le otorguen y los estándares de comparación.

Zapf(1984), con relación a las discrepancias que pudiesen encontrarse entre las evaluaciones "objetivas" y "subjetivas", propone una clasificación cuádruple de conceptos de prosperidad (Tabla 2), aunque esta taxonomía pudiera no ser útil en la práctica, ya que se centra en la evaluación más que en la esencia (Veenhoven, 2000).

El año 1995, Felce \& Perry proponen un cuarto modelo teórico, agregando la necesidad de considerar los valores personales, aspiraciones y expectativas.

\section{d) $C V=$ (Condiciones de vida + Satisfacción con la Vida) * Valores}

Para estos autores, la CV es definida como un estado de bienestar general que comprende descriptores objetivos y evaluaciones subjetivas de bienestar físico, material, social y emocional, junto con el desarrollo personal y de actividades, todas estas mediadas por los valores personales. Bajo este concepto, cambios en los valores, en las condiciones de vida o en la percepción, pueden provocar cambios en los otros, bajo un proceso dinámico.

En esta categoría de definiciones podría incluirse la planteada por la OMS, que en un intento de lograr estudios comparativos e internacionales desarrollaron un grupo de trabajo en CV (World Health Organization Quality Of Life - WHOQOL), el cual propuso una definición propia de CV (The WHOQOL Group, 1995).

Previa a la definición, los investigadores explicitaron algunas de las características del constructo, tales como que (a) la CV es subjetiva. Se distingue la percepción de condiciones objetivas (por Ej. Recursos materiales) y

Tabla 2. Clasificación del concepto de prosperidad - Zapf 1984.

\begin{tabular}{lcc}
\hline & Evaluación subjetiva de bienestar positiva & Evaluación subjetiva de bienestar negativa \\
\hline Evaluación objetiva de bienestar positiva & BIENESTAR & DISONANCIA \\
Evaluación objetiva de bienestar negativa & ADAPTACIÓN & PRIVACIÓN \\
\hline
\end{tabular}


Figura 1. Diagrama del modelo conceptual de la OMS.

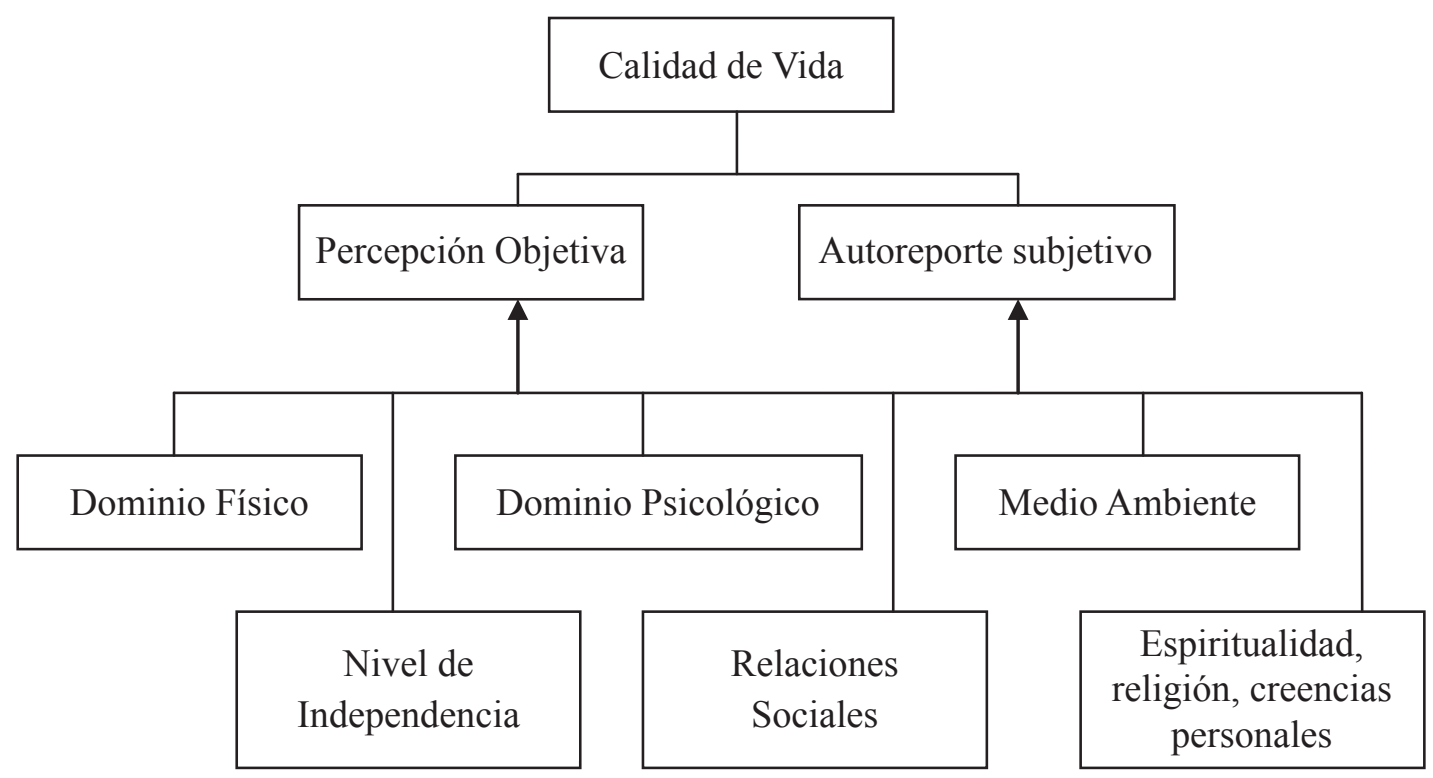

condiciones subjetivas (satisfacción con ese recurso) (b) la naturaleza multidimensional de la CV e (c) incluye tanto funcionamientos positivos como dimensiones negativas (The WHOQOL Group, 1995).

Bajo este marco, la CV es definida como la percepción individual de la posición en la vida en el contexto de la cultura y sistema de valores en el cual se vive y su relación con las metas, expectativas, estándares e intereses.

Aun cuando no lo plantean expresamente, el modelo planteado por la Organización Mundial de la Salud, a través del grupo WHOQOL, puede ser diagramado tal como se observa en la figura 1 .

Esta definición conceptual diferencia claramente las evaluaciones de la persona de su funcionamiento objetivo, capacidades y conductas y los autoreportes subjetivos, en términos de satisfacción / insatisfacción, con estas conductas, estado o capacidades evaluadas en las seis dimensiones propuestas. Cada una de estas dimensiones, a su vez, se compone de subdominios (The WHOQOL Group, 1995). Este modelo presentado por la OMS no es el único, ni ha logrado el consenso de todos los investigadores. Pese a esto, cabe destacar la importancia que en este concepto se otorga a la influencia que ciertos factores tienen en la percepción individual, tales como las metas, las expectativas, estándares e intereses, los que estarían directamente relacionados con la evaluación que se haga en el área y a los factores que en ella inciden, siendo por ende, más que los valores, el factor principal modulador de la evaluación de la $\mathrm{CV}$, el proceso cognitivo de la evaluación.

Tal como se puede apreciar, no existen criterios únicos para definir la $\mathrm{CV}$, sin embargo, aparecen como patrones comunes palabras tales como bienestar, satisfacción, multidimensional, subjetivo/objetivo, que parecen dar un marco común a la diversidad de definiciones, pudiéndose llegar a una definición global de CV como el nivel percibido de bienestar derivado de la evaluación que realiza cada persona de elementos objetivos y subjetivos en distintas dimensiones de su vida. Al ser una medida de autorreporte se centra preferentemente en la evaluación que una persona hace de su nivel de bienestar y satisfacción. El resultado final de esta evaluación es producto de una diversidad de agentes que actúan en la percepción que un individuo tiene sobre su CV, constituyéndose en un abordaje multidimensional, ya que se conjugan diversas valoraciones que hace el propio sujeto sobre las distintas aristas de su vida y de qué manera percibe bienestar o malestar referente a ellas. Bajo este considerando, proponemos un modelo centrado fundamentalmente en el proceso de evaluar qué realiza cada persona.

\section{e) $C V=$ (Condiciones de vida + Satisfacción con la Vida) * Evaluación}

El nivel de CV percibido por un individuo estaría fuertemente modulado por los procesos cognitivos vinculados a la evaluación que la persona realiza tanto de sus condiciones objetivas de vida, como del grado de satisfacción con cada una de ellas.

Ya el año 1999, en un artículo destinado a realizar un meta-análisis para diferenciar CV de estado de salud, Smith, Avis \& Assman (1999) plantearon que la percepción de la CV estaría basada en un proceso cognitivo similar al que se usa para formular actitudes y juicios. Este proceso involucraría (1) identificar los dominios relevantes que la 
comprenden, (2) determinar los estándares en cada dominio e (3) integrar los juicios separados de cada uno de los dominios en una evaluación global de calidad de vida. Bajo esta mirada, la CV es multidimensional, ya que puede incorporar distintas dimensiones para llegar a una sola evaluación.

En este supuesto, la CV aparece como un constructo latente derivado de otros constructos latentes (dominios) y que se expresa en constructos observables (puntajes de evaluaciones). Así, podemos observar un dominio latente a través de uno manifiesto. Las evaluaciones globales pueden corresponder, por ejemplo, a escalas de satisfacción con la vida, mediciones del sentirse bien o bien ítems referidos específicamente a calidad de vida. El puntaje global se correlaciona a través de la dependencia común del factor $\mathrm{CV}$.

Entre los agentes que modulan la percepción, el proceso de comparación se constituye en un elemento esencial, pues los estándares y referencias que se utilicen van a modelar el tipo de respuesta y la valoración otorgada a cada uno de los dominios evaluados (Norman, 2003).

Campbell (1981) propone que las personas juzgan su situación objetiva en cada uno de los dominios evaluados de acuerdo a estándares de comparación basados en aspiraciones, expectativas, sentimientos o lo que podría ser justo tomando como referencia a un grupo de comparación, necesidades personales y valores. Para este autor, el resultado de esta evaluación sería la satisfacción con el dominio evaluado, produciendo las distintas satisfacciones en los dominios un sentimiento general de bienestar. Bajo este considerando, la $\mathrm{CV}$ resulta de la discrepancia entre un estado ideal y uno real, siendo la CV alta cuando la discrepancia es pequeña, y baja cuando ésta es alta (Felce \& Perry, 1995).

En el contexto de identificar procesos cognitivos subyacentes a la evaluación de la CV, Skevington, O`Conell \& WHOQoL Group (2004) desarrollan cuatro posibles opciones que tendrían implícito el proceso de comparación y de valoración por parte de los sujetos. Para estos autores, el evaluar positivamente o no a la $\mathrm{CV}$ dependerá de la relación establecida entre dos factores, por un lado la valoración del dominio como tal, la cual lleva implícita la comparación, y por otro lado la importancia que la persona otorgue a este dominio. Bajo este marco, la evaluación sería un proceso permanente y cambiante, pues los estándares o patrones de comparación utilizados pueden variar en el tiempo, otorgando así a la $\mathrm{CV}$ un carácter dinámico.

El proceso de comparación podría darse en dos sentidos, por un lado comparando el estado actual percibido con un estado ideal, o bien comparándolo con otro estado. En este último tipo de comparación, la persona podría usar como estándares distintas medidas de tiempo (antes - ahora después) o de sujeto (conmigo mismo o con los demás). El cruce de todas estas comparaciones, o fundamentalmente, de la brecha percibida en el tipo de comparación, sería uno de los principales determinantes al momento de evaluar la
$\mathrm{CV}$, ya que el rango de la brecha determinaría el grado de satisfacción (a mayor brecha, mayor insatisfacción), y por ende, el nivel de bienestar por cada dominio.

Diversas teorías han aportado al estudio de la comparación desde diversas aristas. Michalos (1985), cuyos estudios podríamos clasificar en la línea de investigar las brechas percibidas, propone su teoría de las discrepancias múltiples, la cual plantea 6 hipótesis básicas. Entre éstas, sugiere que el reporte de satisfacción está en función de las discrepancias percibidas entre lo que uno tiene y quiere, relevantes para otro, lo mejor que uno tuvo en el pasado, expectativas a tener, lo que se merece y lo que necesita. Asimismo, todas las discrepancias, satisfacción y acciones están directamente e indirectamente afectadas por el sexo, edad, educación, raza, ingreso, autoestima y apoyo social.

La idea de que la satisfacción percibida está en función entre lo que uno tiene y lo que uno quiere tener ha generado varias investigaciones. Una propuesta interesante en este ámbito es la de $\mathrm{Wu}$ (2008), quien propone que al acortar la brecha entre los dominios de la vida que tienen baja discrepancia entre tengo y quiero tener y los dominios con alta discrepancia en tengo y quiero tener, se podrían mejorar los puntajes de autoreporte en CV.

La discrepancia en los niveles de satisfacción ha sido reportado también por Solberg et al., (Solberg, Diener, Wirtz, Lucas \& Oishi, 2002), quienes plantean que la satisfacción es predicha por el grado de discrepancia entre lo que las personas tienen y las que ellos desean, por lo que la posibilidad de alcanzar los bienes deseados desempeña un papel fundamental en la determinación de la satisfacción. Otro estudio realizado por $\mathrm{Wu}$ (2008) sugiere que la satisfacción está asociada con la brecha entre el deseo y la realidad, por lo que ambas investigaciones relacionan a la satisfacción con la discrepancia mencionada, lo que implica que la gente evalúa de manera implícita la distancia de esta brecha de su discrepancia al hacer una evaluación de su satisfacción, factor por tanto trascendental al estudiar los factores mediadores en la percepción subjetiva de la CV realizada por los sujetos.

Desde el punto de vista temporal, ya en el año 1977 Albert plantea la Teoría de la Comparación Temporal, la que plantea que una persona se puede comparar en distintos puntos de tiempo. Es decir, un solo individuo puede comparar una descripción de sí mismo con una descripción de sí mismo en el pasado o en el futuro. Pese a la gran variabilidad que esto pudiese expresar en términos de categorías de evaluación, este mismo autor sugiere una cierta continuidad en las evaluaciones del sujeto dada la necesidad de establecer y mantener un sentido de identidad personal a través del tiempo, otorgando un sentido de continuidad memorial.

Tal como se ha planteado hasta ahora, al momento de compararse la persona podría usar como estándares distintas medidas de tiempo o de sujeto, pudiendo compararse consigo mismo o con los demás. Bajo esta última línea de 
discusión es posible encontrar elementos que aporten a la reflexión desde la teoría de la comparación social.

Aunque teóricamente ha sido estudiada por diversos autores, no es hasta Festinger (1954) que se introduce el término comparación social, el cual hace referencia a las comparaciones que realiza un individuo de sus opiniones y habilidades en relación a las de su grupo, lo que le permite evaluar el propio ajuste a nuevas situaciones.

Festinger (1954) plantea diversas hipótesis con respecto al proceso de comparación social, postulando que existe en el ser humano un impulso a evaluar sus opiniones y sus capacidades. En muchas instancias, evaluar si una opinión es correcta no puede ser inmediatamente determinado por referencias al mundo físico, por lo que el objetivo de estas evaluaciones pueden ser otras personas. Esta necesidad de compararse, como postulan Gilbert, Price \& Allan (1995), es filogenéticamente muy antigua, biológicamente muy poderosa y reconocible en muchas especies. Así también es relevante como base de estudio a los niveles de cognición, conductual y de los procesos afectivos de la vida psíquica del sujeto, influyendo en el autoestima y el bienestar subjetivo. Estas comparaciones parecieran ser la mayoría de las veces un proceso inconciente (Buunk, Belmonte, Peiró, Zurriaga \& Gibbons, 2005), pero podrían determinar el tipo de respuesta que la persona puede generar frente a un determinado suceso (evaluar su estado de salud, por ejemplo).

Tal como plantean Mette \& Smith (1977), es una teoría sobre nuestras preguntas para conocernos a nosotros mismos, sobre la búsqueda de información autorelevante $\mathrm{y}$ cómo las personas ganan autoconocimiento y descubren la realidad sobre sí mismos en ausencia de referentes de realidad objetiva. En la teoría de la Comparación Social, las opiniones y las habilidades de un individuo constituyen una auto-descripción de tal persona en un momento en el tiempo, en comparación con otro.

Las comparaciones son esenciales para la autoevaluación, pudiendo ser empleadas para recopilar información sobre atributos altamente valorados, las expectativas sociales y las normas, de modo que las comparaciones pueden ser realizadas con distintas finalidades, siendo clave el contexto social, donde el grupo de pares participa en un proceso dinámico en el que dedican a compararse y ser objeto de comparación para otros (Jones, 2001).

Este proceso debe considerar el sentido hacia donde se dirige. Puede tener un carácter ascendente, producido al compararse con individuos en mejor situación, o descendente por el contrario. Ambas direcciones pueden influir de distintas maneras, tanto en el estado anímico, en las formas de afrontamiento, en la motivación, en la satisfacción personal, o provocando emociones negativas de envidia, frustración, entre otras, ya que los sujetos basan la valoración que realizan de los distintos aspectos de su vida en relación a la de los demás. En este marco, podemos considerar el proceso de comparación social como provocador de diversas respuestas afectivas, elaborando y construyendo satisfacción o disconformidad con la vida, al evaluar el sujeto si en esta ha alcanzado bienestar o no en relación a lo que observa y evalúa de la vida de otros, lo que inevitablemente se verá reflejado en la construcción que cada uno haga en la evaluación de su CV. Tal como lo plantean Buunk, Kuyper \& Van der Zee (2005), una respuesta negativa hacia los sentimientos de comparación social puede llevar a la frustración y carencia de ambición.

Los resultados de la comparación pueden además estar mediados por la variable compleja de la interpretación, del modelo de identificación-contraste de Buunk \& Ybema (1997), según el cual al compararse socialmente los individuos no solamente pueden contrastarse, sino además identificarse con quienes son sus objetos o sujetos de comparación. Para estos autores, el grado de identificación o similitud viene dado o por la cercanía o por pensar al modelo de comparación como un ejemplo del propio funcionamiento o expectativas futuras, y por otro lado, el contraste se determina también para evaluar el funcionamiento actual del sujeto, se percibe diferencia o distancia.

Debido a estas diferencias en la disposición a la comparación es que se ha comenzado a teorizar sobre las diferencias individuales que median e influyen en el proceso de comparación. En este sentido, Gibbons \& Buunk (1999) han propuesto el concepto de orientación a la comparación social (OCS) como un factor modulador de la comparación social, refiriéndose a la tendencia de algunos individuos a compararse con mayor frecuencia con los demás, diseñando incluso una escala para evaluar esta característica. La OCS expresaría una orientación a tomar en cuenta los sentimientos y respuestas de los demás y una inclinación a acomodarse a los otros. Los individuos con una alta OCS se caracterizarían por una gran preocupación o tendencia a relacionar a sí mismos con otros, estando interesados en los comportamientos y pensamientos de los demás en circunstancias similares. Estudios iniciales indican una relación entre la variable OCS con los dominios social, físico y psicológico de la CV, no obstante, la varianza explicada de estos dominios por la OCS es baja (Urzúa, Castillo, Gemmel \& Campos, 2010). Pese a esto, algunos estudios han referido posibles consecuencias en la esfera afectiva y en el bienestar psicológico derivado de las posibles formas de compararnos con otros (Buunk, Collins, Dakof, Taylor \& VanYperen, 1990; Buunk, Gibbons, Belmonte, Peiró \& Zurriaga, 2005; Buunk, Ybema, Gibbons \& Ipenburg, 2001; Michinov, 2007). En este sentido, aquellos que tienden a compararse hacia arriba presentan mayores efectos negativos en sus afectos (Buunk, 2006; Buunk, Peiró, Rodríguez $\&$ Bravo, 2006), en tanto aquellos que se comparan hacia abajo, podrían generar mayor efecto positivo, especialmente cuando la persona que se compara está sometida a estrés o morbilidad (Buunk, 2006; Buunk \& Ybema, 1997; Friedswijk, Buunk, Steverink \& Slaets, 2004; Gibbons \& 

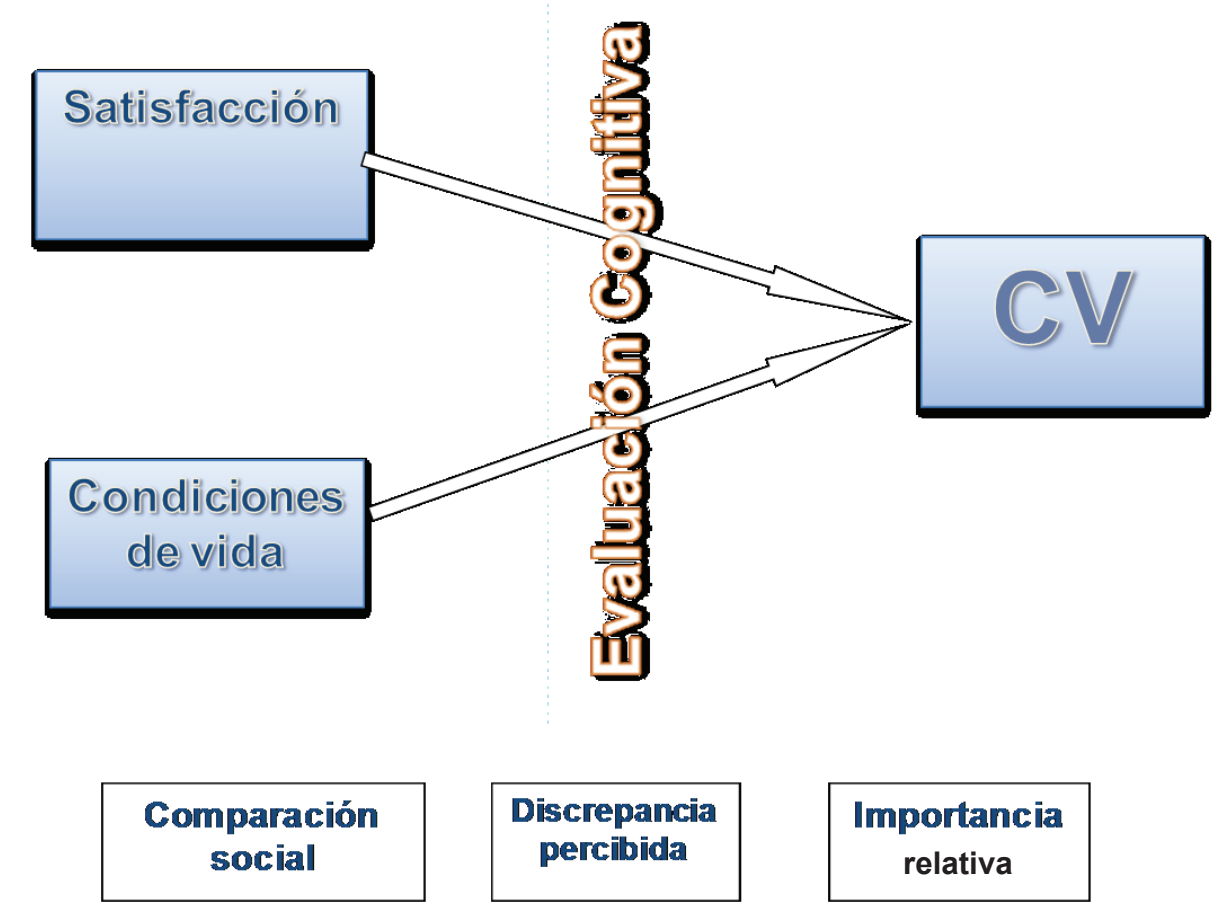

Figura 2: Modelo de Calidad de vida centrado en la evaluación

Buunk, 1999; Gibbons, Gerrard, Lando \& McGovern 1991; Taylor, Wood \& Lichtman, 1985; Van der Zee, Buunk \& Sanderman, 1998; Van Yperen, Brenninkmeijer \& Buunk, 2006; Wheeler \& Miyake, 1992).

Bajo estos considerandos, creemos que el resultado de la evaluación de la $\mathrm{CV}$ estaría mediado por dos grandes procesos, el de la asignación de la importancia que otorgaría cada persona a cada dominio a evaluar, y por otra parte, por el tamaño de la brecha derivado del proceso de comparación, la cual puede ser intrasujeto o extrasujeto. En la comparación intrasujeto jugaría un rol importante las discrepancias (entre lo que quiero/tengo; actual/ideal; pasado/presente/futuro). En la comparación extrasujeto tendría un rol clave la comparación social, y entre estas, la orientación a la comparación social (Figura 2).

\section{f) Otras clasificaciones}

Desde una óptica distinta, aunque complementaria, Ferrans (1990a) sugiere que las aproximaciones a la CV han seguido por dos rumbos: (a) la vida normal y (b) evaluativas. La primera da cuenta de cuan bien funciona un individuo dentro de la sociedad a través de indicadores observables y en la segunda cómo la persona percibe aspectos particulares de su vida, utilizándose para ello variados instrumentos (Bradley et al., 2010).

Tabla 3. Modelo Calidad de Vida - Veenhoven 2000.

\begin{tabular}{lcc}
\hline & Calidades Externas & Calidades Internas \\
\hline Oportunidades de vida & Habitabilidad del entorno & Capacidad para la vida del individuo \\
Resultados de vida & Utilidad de vida & Apreciación de vida \\
\hline
\end{tabular}

Tabla 4. Ejemplos Modelo Calidad de Vida - Veenhoven 2000.

\begin{tabular}{lll}
\hline & Calidades Externas & Calidades Internas \\
\hline \multirow{2}{*}{ Oportunidades de vida } & Aire limpio & Salud física \\
& Seguridad social & Salud mental \\
\multirow{2}{*}{ Resultados de vida } & Criar hijos & Satisfacción con la vida \\
& Ser buen ciudadano & Nivel de humor \\
\hline
\end{tabular}


Ferrans (1990a) señala que se pueden encontrar definiciones focalizadas en la habilidad de funcionamiento, en la felicidad o la satisfacción, y otros en el cumplimiento de metas, utilidad social o capacidades.

Veenhoven (2000) propone una categorización distinta en que combina la diferencia existente entre lo potencial y lo real, así como con lo interno y lo externo (Tabla 3).

Por habitabilidad del entorno, se refiere explícitamente a las características del entorno, denominado por alguno nivel de vida (o prosperidad para los economistas). Por capacidad para la vida del individuo considera a las oportunidades internas de vida, es decir, el cómo se está preparado para afrontar los problemas, lo que en biología se denomina potencial de adaptación. Por utilidad de vida, parte del supuesto de que la vida buena tiene que ser buena para algo más que por sí misma, es el resultado del hecho que una persona es útil en algún aspecto. Finalmente, por apreciación de vida, se refiere a la apreciación subjetiva de la vida, calificada generalmente como bienestar subjetivo, satisfacción con la vida y felicidad. La Tabla 4 ejemplifica los componentes del modelo.

Spilker (1990) plantea un modelo jerarquizado de CV, compuesto por tres niveles que van desde la CV general (nivel uno), pasando por las dimensiones o dominios que componen la CV (nivel dos), hasta los aspectos específicos de cada dominio. Bajo este supuesto, la definición de CV propuesta por la OMS pertenecería al nivel 1 y los dominios que la comprenden al nivel 2.

Este modelo asume que las variables de más bajo nivel determinan los niveles superiores, es decir, bajas puntuaciones en el nivel tres o dos, determinarían una mala evaluación de la CV general (nivel 1), lo cual no ha sido comprobado.

Para Arnold et al., (2004), esta distinción en niveles es relevante, ya que la medición del estado funcional provee información detallada acerca de las consecuencias de una enfermedad y permite evaluar a qué dominios y en cuánto ha afectado la enfermedad, por otra parte, la percepción general de $\mathrm{CV}$ puede ser más resistente al cambio y por lo tanto menos aplicable de medir cambios en la condición de paciente fruto de una enfermedad.

Cummins (2000) reafirma esta idea. Para él, las personas que tienen la habilidad de adaptarse a los factores cambiantes intentarán mantener un nivel estable de bienestar, y por otro lado, los dominios de CV probablemente no cubran toda la $\mathrm{CV}$ de la persona. Por otra parte, la evaluación de la $\mathrm{CV}$ general requiere que las personas evalúen los diversos dominios y combinen el peso que le dan a cada uno de estos con un juicio valórico genérico.

\section{Discusión}

Pese a la gran cantidad de investigaciones que en la actualidad es posible encontrar sobre $\mathrm{CV}$ y fundamentalmente sobre CV relacionada con la salud, aún es posible encontrar líneas de discusión que podrían afectar el desarrollo de esta área de estudio.

\section{Diferenciación con otros conceptos:}

Tal como ya se ha planteado, es necesario distinguir CV de otros conceptos, como estado funcional, satisfacción con la vida, bienestar o estado de salud, pues a menudo investigaciones en esas áreas son interpretadas como mediciones en CV (Haas, 1999). Como una manera de enfrentar este dilema, partidarios de dividir el constructo $\mathrm{CV}$ en sub áreas específicas, han propuesto reducir la confusión entre éste y los factores que contribuyen a ella, evitando así problemas en identificar resultados de tratamientos o variables de la enfermedad (Taillefer, Dupuis, Roberge \& Lemay, 2003). Esta especificidad de la evaluación de la CV en áreas concretas de la vida de las personas, ha permitido el desarrollo de conceptos secundarios, como lo es la CV relacionada con la salud, al referirnos al nivel de bienestar derivado de la evaluación que la persona realiza de diversos dominios de su vida, considerando el impacto que en éstos tiene su estado de salud (Urzúa, 2010).

\section{Evaluación transversal versus longitudinal:}

El dilema acerca de la validez de la medición transversal de la CV frente a evaluaciones de tipo longitudinal, partiendo del supuesto de que el constructo $\mathrm{CV}$ es dinámico, es un tópico que aún no tiene una respuesta definitiva, aun cuando la mayoría de las investigaciones en CV son realizadas con un tipo de medición de corte, es decir, transversal y no consideran el cambio en la percepción originado con el paso del tiempo o la modificación de los factores que la modulan.

Allison, Locker \& Feine (1997) sostienen que frecuentemente en las evaluaciones se consideran sólo las diferencias intersujetos, pero no las intrasujetos, dadas por el carácter dinámico de este constructo, lo que sería posible de realizar bajo un modelo longitudinal

\section{Otros dilemas}

Fernández-Ballesteros (1998) resume las principales líneas de discusión en torno al concepto de calidad de vida fundamentalmente en dos: (a) $\mathrm{Si}$ la $\mathrm{CV}$ corresponde netamente a una percepción subjetiva de la persona sobre ciertas condiciones, o bien, incluir además de esta percepción, la evaluación objetiva de las mismas condiciones y (b) si la CV se refiere a un concepto ideográfico (el propio sujeto establece sus componentes) o bien nomotético (estableciendo un criterio general para todos los sujetos). Otros problemas vinculados a la investigación en CV son (a) inexistencia de un modelo o marco teórico que avale la definición presentada, (b) no diferenciación con factores 
que influencian la $\mathrm{CV}$ y (c) la validez de los instrumentos de medición utilizados.

\section{Algunos acuerdos}

Pese a los problemas pendientes, para Schalock (2000) la investigación en CV en los años 90 ha comenzado a responder dilemas tales como: ¿la CV es un concepto único?, ¿Es el constructo CV unidimensional o multidimensional?, y si fuera unidimensional, ¿sería el mismo para todos los individuos al estar basado en evaluaciones subjetivas?, dada su naturaleza, ¿puede ser medida?, ¿cómo?, y ¿bajo qué estándares psicométricos? (Sawatzky, Ratner \& Chiu, 2005).

En esta misma línea de proponer acuerdos comunes, Meeberg (1993) sintetiza elementos de una revisión de la literatura y enumera cuatro atributos críticos para diferenciar CV de otros conceptos: (a) Sentimiento de satisfacción con la vida en general, (b) capacidad mental para evaluar la vida propia como satisfactoria o no, (c) un aceptable estado de salud físico, mental, social y emocional determinado por los sujetos y (d) una evaluación objetiva realizada por una persona sobre las condiciones de vida.

A manera de conclusión, y tomando lo planteado por Fernández-Ballesteros (1998), se puede decir que la literatura sobre $\mathrm{CV}$ concuerda fundamentalmente en tres cosas: (a) Es subjetiva, (b) el puntaje asignado a cada dimensión es diferente en cada persona y (c) el valor asignado a cada dimensión puede cambiar a través de la vida. En este contexto es importante destacar la necesidad que plantean algunos autores de incorporar aspectos evolutivos en la evaluación específica de la calidad de vida (Trujillo, Tobar \& Lozano, 2004).

\section{Referencias}

Albert, S. (1977). Temporal comparison theory. Psychological Review, 84, 485-503.

Allison, P., Locker, D., \& Feine, J., (1997). Quality of life: A dynamic construct. Social Sciences and Medicine, 45, $221-230$.

Arnold. R., Ranchor, A., Sanderman, R., Kempen, G., Ormel, J., \& Suurmeijer, T. (2004). The relative contribution of domains of quality of life to overall quality of life for different chronic diseases. Quality of life Research,13, 883-896.

Atkinson, M., Zibin, S., \& Chuang, H. (1997) Characterizing quality of life among patients with chronic mental illness: A critical examination of the self-report methodology. American-Journal of Psychiatry, 154, 99-105.

Bigelow, D. A., McFarland, B. H., \& Olson, M. M. (1991). Quality of life of community mental health program clients: Validating a measure. Community Mental Health Journal, 27, 43-55.

Bishop, S., Walling, D., Dott, S., Folkes, C., \& Bucy, J., (1999). Refining quality of life: Validating a multidimensional factor measure in the severe mentally ill. Quality of life Research, 8, 151-160.

Bognar, G. (2005). The concept of quality of life. Social and Practice, 31, 561-580.

Borthwick-Duffy, S. A. (1992). Quality of life and quality of care in mental retardation.

In L. Rowitz (Ed.), Mental retardation in the year 2000 (pp. 52-66). Berlin: Springer Verlag.
Bradley, J., Thorlund, K., Schünemann, H., Xie, F., Hassan, M., Montori, V., \& Guyatt, G. (2010). Improving the interpretation of quality of life evidence in meta-analyses: The application of minimal important difference units. Health and Quality of Life Outcomes, 8,116.

Buunk, A. (2006). Responses to a happily married other: The role of relationship satisfaction and social comparison orientation. Personal Relationships, 13, $397-409$.

Buunk, A., Belmonte, J., Peiro, J., Zurriaga, R., \& Gibbons, F. (2005). Diferencias individuales en la comparación social: Propiedades de la escala española de orientación hacia la comparación social. Revista Latinoamericana de Psicología, 37, 561-579.

Buunk, A., Collins, R., Taylor, S., VanYperen, N., \& Dakof, G. (1990). The affective consequences of social comparison: Either direction has it ups and downs. Journal of Personality and Social Psychology, 59, $1238-1249$.

Buunk, B., Kuyper, H., \& Van der Zee, Y. (2005). Affective response to social comparison in the classroon. Basic and applied social psychology, 27, $229-237$.

Buunk, B., Nauta, A., \& Molleman, E. (2005). In search of the true group animal the effects of affiliation orientacion and social comparision orientation upon group satisfaction. European Journal of personality, $19,69-81$.

Buunk, A. P., Peiró, J.M., Rodríguez, I., \& Bravo, M.J. (2007). A loss of status and a sense of defeat: An evolutionary perspective on professional burnout. European Journal of Personality, 21, 471-485.

Buunk, B., \& Ybema, J. (1997). Social comparisons y occupational stress: The identification-contrast model. En B. P. Buunk \& F. X. Gibbons (Eds.), Health, coping, $y$ well-being: Perspectives from social comparison theory (pp. 359-388). Mahwah, NJ: Erlbaum

Buunk, A., Ybema, J., Gibbons, F., \& Ipenburg, M. (2001). The affective consequences of social comparison as related to professional burnout and social comparison orientation. European Journal of Social Psychology, 31, $337-351$.

Calman, K.C. (1987). Definitions and dimensions of quality of life. En N.K. Aaronson \& Beckman (Eds.), The Quality of life cancer patients (pp.1-9). New York: Ravens Press.

Campbell, A. (1981) The sense of well-being in america. McGraw-Hill, New York.

Campbell A., Converse P., \& Rodgers W. (1976) The Quality of American Life. New York: Russell Sage.

Caqueo-Urízar, A., Gutiérrez-Maldonado, J., \& Miranda-Castillo, C. (2009). Quality of life in caregivers of patients with schizophrenia: A literature review. Health and Quality of Life Outcomes, 7, 84.

Caqueo-Urízar, A., \& Lemos-Giráldez, S. (2008). Calidad de vida y funcionamiento familiar en pacientes con esquizofrenia, en una comunidad Latinoamericana. Psychothema, 20, 577-582.

Cummins, R.A. (2000). Objective and subjective quality of life: An interactive model. Social Indicators Research, 52, 55-72

Cummins, R. A. (2004). Moving from the quality of life concept to a theory. Journal of Intellectual Disability Research, 49, 699-706.

Edgerton, R. B. (1990). Quality of life from a longitudinal research perspective. In R. L. Schalock (Ed.), Quality of life: Perspectives and issues (pp. 149-160). Washington DC: American Association on Mental Retardation.

Felce, D., \& Perry, J. (1995). Quality of life: Its definition and measurement. Research in developmental disabilities, 16, 51-74.

Fernández-Ballesteros, R. (1998). Quality of Life: The differential conditions. Psychology in Spain, 2, 57 - 65.

Ferrans, C. (1990a) Quality of Life: Conceptual Issues. Seminars in Oncology Nursing, 6, $248-254$.

Ferrans C. E. (1990b). Developement of a quality of life index for patients with cancer. Oncology Nursing, 17, $15-21$.

Festinger, L. (1954). A theory of social comparison processes. Human Relations, 7, 117-140.

Frieswijk, N., Buunk, B., Steverink, N., \& Slaets, J. (2004). The interpretation of social comparison an its relation to life satisfaction among elderly people: Does frailty make a difference? Journal of Gerontology: Psychological Sciences, 59B, $250-257$. 
Gibbons, F., \& Buunk, B. (1999). Individual differences in social comparison: Development of a scale of social comparison orientation. Journal of Personality and Social Psychology, 76, 129 - 142.

Gibbons, F., \& Buunk, B. (1999). Individual differences in social comparison: Development of a scale of social comparison orientation. Journal of Personality and Social Psychology, 76, 129 - 142.

Gilbert, P., Price, P., Allan, S., (1995) Social Comparison, social attractiveness and evolution: How might they be related? New Ideas in Psychology, 13, 149-165.

Gill, T.M., \& Feinstein, A.R. (1994). A critical Appraisal of the quality of life measurements (abstract). Journal of the American Medical Association, 272, $619-626$.

Haas, B. (1999). Clarification and integration of similar quality of life concepts. Journal of nursing Scholarships, 31, 215 - 220.

Hollanflsworth J.G. Jr. (1988) Evaluating the impact of medical treatment on the quality of life: A 5-year update. Social Science and Medicine, 26, 425-434.

Hornquist J.O. (1982) The concept of quality of life. Scandinavian Journal of Social Medicine. 10, 57-61.

Huppert, F., \& Whittington, J., (2003). Evidence for the independence of positive and negative well - being : Implications for quality of life. British Journal of Health psychology, 8, 107 - 122.

Jenkins C.D. (1992) Assessment of outcomes of health intervention. Social Science \& Medicine, 35, 67-375.

Jones, D. (2001). Social comparison and body image: Attractiveness comparison to models and peers among adolescents girls and boys. Sex roles, 45, $645-664$.

Lawton, M. (1999). Quality of life in chronic illness. Gerontology, 45, 181-183.

Lefley, H.P. (1998). Foreword quality of life model. Community Mental Health Journal. 34, 455 - 457.

Martin, A., \& Stockler, M. (1998). Quality-of-life assessment in health care research and practice. Evaluation and the Health Professions, 21, 141-156.

Meeberg, G.A. (1993). Quality of life: A concept analysis. Journal of Advanced Nursing, 18, $32-38$.

Mettee, D. R. \& Smith, G. (1977). Social comparison and interpersonal attraction: The case for dissimilarity. En J. M. Suls \& R. L. Miller (Eds.), Social comparison processes: Theoretical and empirical perspectives (pp. 21-41). Washington, DC: Hemisphere.

Michalos, A. (2004). Social Indicators research and health related quality of life research. Social Indicators Research, 65, 27 - 72.

Michalos, A. (1985). Multiple discrepancies theory. Social Indicators Research, 16, 347-413.

Michalos, A. (2004). Social indicators research and health-related quality of life research. Social Indicators Research, 65, 27-72.

Michinov, N. (2007). Social comparison and affect: A study among elderly Women. The Journal of Social Psychology, 147, 175 - 189.

Norman, G. (2003) Hi! How are you? Response shift, implicit theories and differing epistemologies. Quality of Life Research, 12, 239-249.

O'Boyle, C. (1994) The Schedule for the evaluation of individual Quality of life (SEIQoL). Internacional Journal of Mental Health, 23, 3 - 23.

Oppong J.R., Ironside R.G., \& Kennedy L.W. (1987) Perceived Quality of Life in a Centre: Periphery Framework. Edmonton Area Series Report No. 52. Department of Sociology, University of Alberta, Edmonton, Alberta. USA.

Sawatzky, R., Ratner, P., \& Chiu, L. (2005). A Meta-analysis of the relationship between spirituality adn quality of life. Social Indicador Research, 72, 153-188.

Schalock, R., (2000) Three decades of quality of life. Focus en Autism and other developmental disabilities, 15, 116.

Shaw A. (1977) Defining the quality of life. Hastings Center Report 7, 11.

Shen, S., \& Lai,Y. (1998) Optimally scaled quality-of-life indicators. Social Indicators Research, 44, 225-254.
Skevington, S., O'Conell, K., \& The WHOQOL Group (2004). Can we identify the poorest quality of life? Assessing the importance of quality of life using the WHOQOL -100. Quality of life Research, 13, 23 - 34.

Smith, K., Avis, N., \& Assman, S. (1999). Distinguishing between quality of life and health satus in quality of life research: A meta-analysis. Quality of life research, 8, $447-459$

Solberg, E., Diener, E., Wirtz, D., Lucas, R., \& Oishi, S. (2002). Wanting, having, and satisfaction: Examining the role of desire discrepancies in satisfaction with income. Journal of Personality and Social Psychology, 83, 725-734.

Spilker, B. (1990) Introduction to the field of Quality of Life trials. En B. Spilker (Ed.), Quality of Life Assessments in Clinical Trials (pp. 3-10). New York: Raven Press.

Taillefer, M.C., Dupuis, G., Roberge, M.A., \& Le May, S. (2003). Healthrelated quality of life models: Sistematic review of the literature. Social Indicators Research, 64, $293-323$.

Taylor, S., Wood, J., \& Lichtman, R. (1985). Social comparison in adjustment to breast cancer. Journal of Personality and Social Psychology, 49, 1169-1183.

The WHOQOL Group, (1995) The World Health Organization Quality of life assessment (WHOQOL): Position paper from the world health organization. Social Science and Medicine, 41, 1403 - 1409.

Trujillo, S., Tobar, C., \& Lozano, M. (2004). Formulación de un modelo teórico de la calidad de la vida desde la psicología. Universitas Psychologica, 3, 89-98.

Urzúa, A. (2010). Calidad de vida relacionada con la salud: Elementos conceptuales. Revista Médica de Chile, 138, 341 - 348.

Urzúa, A., Castillo, P., Gemmel, M., \& Campos, C. (2010). Relación entre la orientación a la comparación social y el autoreporte de calidad de vida. Revista Colegio Psicólogos del Perú, 9, 10 - 30.

Van der Zee, K., Buunk, B., \& Sanderman, R. (1998). Neuroticism and reactions to social comparison information among cancer patients. Journal of Personality, 66, $175-194$.

VanYpereng, N., Brenninkmeijer, V., \& Buunk, B. (2006). People's responses to upward social comparisons: The role of the individual's effort - performance expectancy. British Journal of Social Psychology, $45,519-533$

Veenhoven, R. (2000) The four qualities of life. Ordering concepts and measures of the good life. Journal Of Happiness Studies, 1, 1 - 39.

Verdugo, M., Schalock, R., Keith, K., \& Stancliffe, 2005). Quality of Life and its measurement: Important principles and guidelines. Journal of Intellectual Disability Research, 49, 707-717.

Wahl, A., Hanestad,B., Wiklund, I., \& Moum,T. (1999). Coping and quality of life in patients with psoriasis. Quality of Life Research, 8, 427-433.

Wheeler, L., \& Miyake, K. (1992). Social comparison in everyday life. Journal of Personality and Social Psychology, 62, 760-773.

Wrosch, C., \& Scheier, M. (2003). Personality and quality of life: the importance of optimism and goal adjustment. Quality of life Research, $12,59-72$.

$\mathrm{Wu}, \mathrm{C}$ (2008). The role of perceived discrepancy in satisfaction evaluation. Social Indicators Research, 88, 423 - 436.

Zapf, W. (1984), Individuelle Wohlfahrt: Lebensbedingungen undwahrgenommene Lebensqualität. En W. Glatzer \& W. Zapf. (Eds.), Lebensqualität in derBundesrepublik. Objective Lebensbedingungen und subjectives Wohlbefinden (pp. 13-26). Frankfurt:Campus Verlag. 